\title{
Automatic Segmentation of Dental Cone Beam Computed Tomography Image Based on Level Set Method Using Morphology Operators and Polynomial Fitting
}

\author{
Fahmi Syuhada* and Agus Zainal Arifin \\ Department of Informatics, Faculty of Information and Communication Technology, \\ Institut Teknologi Sepuluh Nopember (ITS) Surabaya \\ Email:fahmi.18051@mhs.its.ac.id,agusza@cs.its.ac.id
}

*Corresponding Author

\begin{abstract}
Automatic Segmentation of dental cone beam computed tomography (CBCT) images is challenging due to the intensity of the teeth that have low level intensity. In this paper we propose a new method for an automatic teeth segmentation in slices of CBCT images based on level set method using morphology operators and polynomial fitting. Morphology operators are used to construct the Region of Interest (ROI) area of dental objects in the image slice. ROI is used to focus the analysis process on areas of dental objects which generally have a polynomial pattern distribution. This is useful for simplifying phi model variables that are generally used in the level set method. Polynomial fitting is obtained to estimate arc of teeth structure in CBCT images. Level set is implemented to evolve the ROI to obtain the contours of dental objects. Comparison between proposed method result and the ground truth images shows that the method gives the best average accuracy, sensitivity, and specificity value of $99.02 \%, 95.32 \%, 99.09 \%$, respectively. These values are promising for accurate segmentation of the entire tooth form on CBCT images.
\end{abstract}

Key words: Dental segmentation, CBCT, Level Set, Morphology Operator, Polynomial fitting.

\section{INTRODUCTION}

The ability of medical images in visualize the human body including internal organs, bones, and teeth has become an important role in medicine as a tool for analysis and diagnosis [1]. Dental Cone Beam Computed Tomography (CBCT) image is one of a variety of medical images that visualizes the shape of the tooth structure in the mouth in 3D images model [2]. Dental CBCT image processing is done by reconstructing it become two Dimension image structure called slice that visualized from

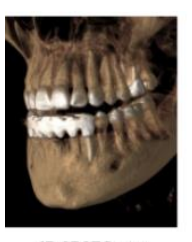

3D CBCT Image
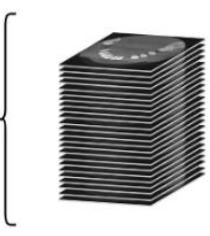

Stack of 2D Images (Slices)

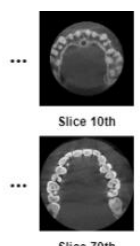

Slice 70th

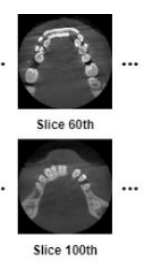

Fig. 1 CBCT representation, Stack of 2D slice, each slice images the axial direction. Fig. 1 shows the CBCT image from 3D data which is then reconstructed into a 2D image slice array from the axial direction. Computer science research is concerned with processing CBCT data using this data models to obtain certain information.

In the past decades, several methods have been proposed to segment teeth from dental CBCT images scanned. Generally, the proposed segmentation method can be classified into two types, namely label propagation and boundary tracking [3]. Segmentation label propagation model is the process of analyzing a coordinate or pixel area of an image [4]. Meanwhile, the Boundary Tracking segmentation model is a segmentation whose process is to find the shape of an object in an image based on its edges [5]. In the latest research, segmentation of dental CBCT image is also done by applying deep learning in the process of detecting objects [6].

Region growing is a type of label propagation model method used [7]. Region growing does segmenting by finding similarities of the sub-area of an area in images based on several properties, including intensity, color, and texture, respectively [8]. Ref. [9] developed segmentation method that utilizes 3D CBCT information using the region merging algorithm to recognize tooth elements that have similar intensity values to other elements. The learning method is also developed by training process with dental CBCT with ground truth as input and target data [6].

The boundary tracking model in segmenting dental CBCT images in the last decade is being widely implemented. The previous model was not optimal because of certain factors. The intensity of the teeth that have low levels and similarities to bone tissue is a factor less than optimal results obtained [10]. Boundary tracking can be used more to overcome this factor compared to the previous model [11]. This segmentation model is finding teeth objects in dental CBCT images based on the shape of the contours. Level set is a segmentation method based on boundary or contour tracking [3], [11], [12].

Level set is a boundary tracking algorithm that is widely used for segmenting dental CBCT images in the last 
decade. This method has advantages in dealing with topological change and contour propagation [13]. Gao and Chae [14] developed the dental CBCT segmentation method based on the level set method with shape and intensity prior to the previous slice to segment the tooth and achieved promising results. Ji et al. [15] developed a level set framework for anterior teeth segmentation. The hybrid level set model was developed which integrated few method to generate optimal results [16]. Yau et al. [17] applied level set method which focuses on reconstruction based on data fusion. Xia et al [13] applied a level set method that focuses on the maxillary and mandible of teeth structure.

Contour initialization is the first step of the level set method. Then the contour will be evolved to find the contour of the object in the initialized area [18]. The Previous tooth segmentation method initialized contours by manually marking each tooth object [13], [14]. Teeth structure in general has a polynomial distribution area pattern. By processing in that area so it will make segment the teeth more optimal. This pattern of dental object region distribution can be obtained so that the segmentation process can be focused on only the region. In addition, this area can also be utilized to form a process segmentation automation process by implementing several morphology operators.

In this paper we propose a new method for automatic segmentation based on level set using morphology operators and polynomial fitting. Morphology operators are used to construct the Region of Interest (ROI) area of dental objects in the image slice. ROI is used to focus the analysis process on areas of dental objects which generally have a polynomial pattern distribution. Polynomial fitting is obtained to estimation arc of teeth structure in CBCT images. Level Set is implemented to evolve the ROI to obtain the contours of dental objects.

This paper is organized as follows. We describe the mathematical morphology operator, polynomial fitting, and level set in detail in Section 2. In Section 3, we present our method for automatic segmentation of the teeth in CT images. In Section 4, some promising results are demonstrated and analyzed. Finally, we conclude our work with a summary of the paper and some future works in Section 5

\section{BACKGROUND}

\section{A. Mathematical Morphology}

Morphology is a domain in image processing that is useful for analyzing the shape of regions in an image such as boundaries and skeletons [19]. There are several morphological operators in image segmentation, such as erosion, dilation, opening, closing, filling, skeleting, etc. Morphological dilation makes objects more visible and fills in small holes in objects. The dilation of $I$ by $G$ denoted as $I \oplus G$ that is defined as Equation (1):

$$
I \oplus G=\left\{z \mid\left(G^{s}\right) z \cap I \neq \emptyset\right\}
$$

where $\varnothing$ is empty set and $G$ is structuring element. Morphological erosion removes islands and small objects so that only substantive objects remain. The erosion $I$ by $G$ is defined as Equation (2):

$$
I \ominus G=\left\{z \mid\left(G^{s}\right) z \cap I^{c} \neq \emptyset\right\}
$$

where $I^{c}$ is the complement of $I$. Morphological opening is useful for removing small objects from an image while preserving the shape and size of larger objects in the image as Equation (3)

$$
I \circ G=(I \ominus G) \oplus G
$$

where $\Theta$ dan $\bigoplus$ denote erosion and dilation, respectively. Morphological closing is useful for filling small holes from an image while preserving the shape and size of the objects in the image as Equation (4).

$$
I \cdot G=(I \oplus G) \ominus G
$$

\section{B. Polynomial Fitting}

Polynomial Fitting is a technique used to find the right curve for a set of points $\left(\mathrm{x}_{1}, \mathrm{y}_{1}\right) \ldots\left(\mathrm{x}_{\mathrm{n}}, \mathrm{y}_{\mathrm{n}}\right)$ of course by minimizing the number of errors of the smallest square of data points of the curve $(x, y)$ formed. There are many curve models that can be formed from a collection of points, but the shape of the curve formed by the CBCT image in each slice is polynomial. LSCF determines the approach function of the polynomial value $y=f(x)$ which is the closest point $\left(\mathrm{x}_{1}, \mathrm{y}_{1}\right) \ldots\left(\mathrm{x}_{\mathrm{n}}, \mathrm{y}_{\mathrm{n}}\right)$. Polynomial fitting is defined as the nth order equation as follows:

$$
f(x)=a_{n} x^{n}+a_{n-1} x^{n-1}+\cdots+a_{1} x^{1}+a_{0} x^{0}
$$

\section{Level Set Method}

Segmentation with a Boundary Tracking approach is a segmentation in the process of tracking the contours of objects in an image. In the current decade, one method that is widely used and developed is the level set method [12]. The level set method is a common numerical method for the curve change process to be evolved in the object tracking process. The Distance Regularized Level Set Evolution (DRLSE) method is the development of the level set method used in this paper [18]. Energy minimization formulation is carried out for the process of contour evolution to trace the shape of the object being segmented. Equation (6) presents the contour energy minimization formula.

$$
\varepsilon(\phi)=\mu \mathcal{R}_{p}(\phi)+\lambda \mathcal{L}_{g}(\phi)+\alpha \mathcal{A}_{g}(\phi)
$$

where $\mathcal{R}_{p}(\phi)$ is a relationship term for level set regulation. $\mathcal{L}_{g}(\phi)$, and $\mathcal{A}_{g}(\phi)$ are terms for some energy that drive the curve movement of the level set function. 


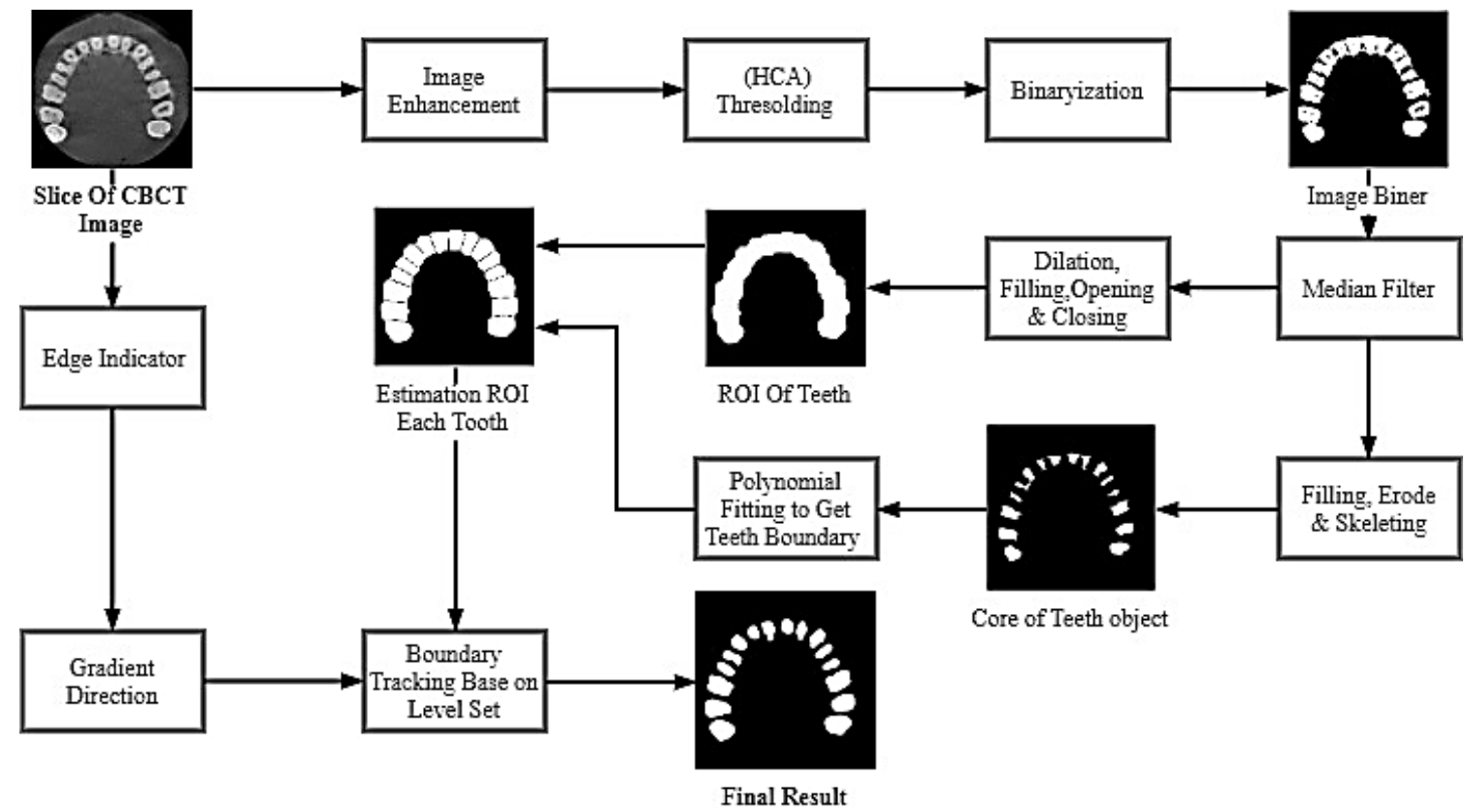

Fig. 2 Diagram of proposed segmentation technique to dental slice CBCT image

\section{PROPOSED METHOD}

In this section, we introduce our proposed method to automatic segmentation of slice dental CBCT image. Generally, our proposed method is shown in Fig. 2. There are various processes carried out from a slice of CBCT image test data until the images result is obtained. However, the process can be divided into several steps as follows:

- First Step: Binarization Slice of dental CBCT test image.

- Second Step: Determining the ROI of teeth area.

- Third Step: Getting the core of dental object and find the centroid of tooth.

- Fourth Step: Estimation boundary of each dental object.

- $\quad$ Fifth Step: Final segmentation

In the remainder of this section, we review each several steps of our proposed method.

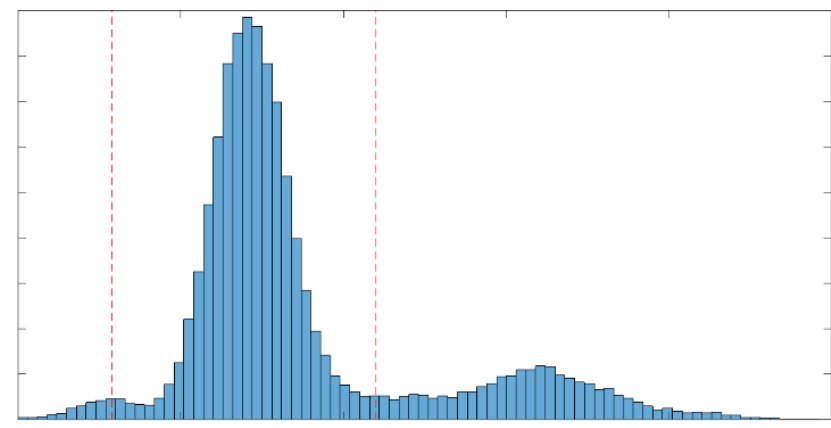

Fig. 3 Clustering of histogram value using HCA method

\section{A. First step: Binarization to slice of dental CBCT test image}

Direct binarization of the image is carried out to obtain the black and white image based on the threshold value. In Fig. 2, this step is begun from image an image enhancement, Hierarchy Cluster Analysis (HCA) thresholding, and the last is binarization. We applied simple enhancements to images such as contrast adjustment and sharpening them. For thresholding process, we implemented HCA method to obtain the value. The HCA method supports the multi-thresholding process to cluster the histogram image value into few group [20]. Fig. 3 shows a histogram diagram of test image that has been divided into three object clusters with two threshold values. We assume that the dental object is in the third cluster because in the dental CBCT image, teeth object area has a higher intensity than the other area. Therefore, we use the second threshold value to be used in the binarization step to obtain a black and white image.

\section{B. Second step: Determining the ROI of teeth area}

Determining of the ROI is used to obtain an area that only contains the dental object. In addition, ROI is used in the next step to construct the estimated tooth area that used as initialization contour in the final segmentation process. In Fig. 2, determination of ROI area is begun from Median filter and Morphological process consisting erosion, dilation, filling, opening, and closing. Median filter is useful for denoising salt and pepper noise in black and white teeth image obtained in the first step. The binarization results in the form of black and white images show the object and background of the dental CBCT slice. Dental objects generally have an almost dominant shape. However, sometimes there is noise that appears. The noise can be in the form of salt and paper noise. Besides that, 


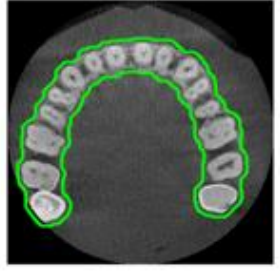

a

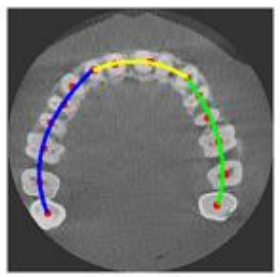

c

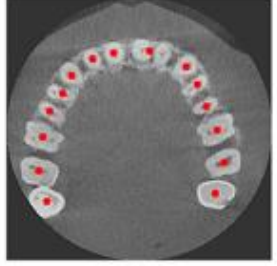

b

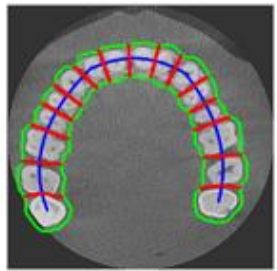

d
Fig. 4 (a) ROI area of teeth distribution. (b) Centroid of each tooth. (c) Kurva polynomial fitting. (d) Boundary of each tooth object

noise that is actually not a dental object will appear. This is because the shape is different and has a smaller size to be said to be a dental object. Erosion process removes the various forms of noise. On the other hand, this process affects the process of erosion of the entire object, especially objects teeth. To reverse the dental objects, the dilation process is carried out. Dilation process using stel value adjustment that returns the object to its original size shape or exceeds the threshold value. Opening is a combination of erosion-dilation with the same structuring element. This operation will remove the white "hole" in a dark (black) object. Closing is a combination of dilation-erosion with the same structuring element. This operation will remove the black "hole" on a bright (white) object. Fig. 4a is the determined ROI Area which shows that all dental objects are in thre area.

\section{Third Step: Getting the core of dental object and find the centroid of tooth object}

In the proposed method, ROI area is not directly used to obtain tooth object. There is a process to further specify the ROI to obtain the dental object area. In Fig. 2 Polynomial fitting curve to get boundary of each teeth object is one wat to process it. The process will be explained in the next section. However, the step that must be done to obtain the polynomial curve is to provide the centroid of each dental object in the image. In Fig. 2, centroids id is obtained from the core of tooth object image. Morphology operator like filling, eroded, and skeleton region props is applied to black and white image from the first step. The idea of the process is how to reduce the binary image until parts of each tooth object area separated from each other. Fig. $4 \mathrm{~b}$ shows the result of centroid determination process from third step.

\section{Fourth Step: Estimation boundary of each dental object using polynomial fitting}

Specification of ROI Area can improve the accuracy of boundary tracking in the final segmentation. Fig. 2 shows the ROI area specifications based on the boundaries of each tooth object boundary. The process begins from the determination of the polynomial fitting curve based on the centroid point that has been obtained in the previous step. Polynomial fittings are used to represent the arc estimation of the tooth structure. Polynomial fittings are used to represent the arc estimation of the tooth structure. To obtain optimal curves, we divided the process of forming curves into three processes. The first curve was formed from the centroid point to one third of the size of the ROI. The next curve was formed from the end point of the previous curve to the process of forming the third curve. Fig. 4c shows three fitting curves form to represent the arc of the tooth structure in the dental CBCT image.

Determination of the boundary of each dental object is based on the calculation of the projection value based on points that form the polynomial curve. The projection value is calculated based on the grayscale value that passes through the projection perpendicular to the coordinates of a polynomial fitting point to the tooth ROI area boundary. The boundary is defined as a point with an average grayscale intensity projected below the thresholding value of the gear object. Fig. 4d shows the result of determining the boundary of each dental object in the ROI area. The implementation of the tooth object boundary is carried out at the ROI shown in Fig. 2.

\section{E. Final Segmentation}

In this step, final segmentation of slice CBCT images is done using Distance Regularized Level Set Evolution (DRLSE) Method. In Fig. 2, the final segmentation consists of edge indicator processes, gradient direction, and boundary tracking of tooth objects. There are two main images processed in this step, the original image and the ROI that has been obtained which is then specified in the previous step.

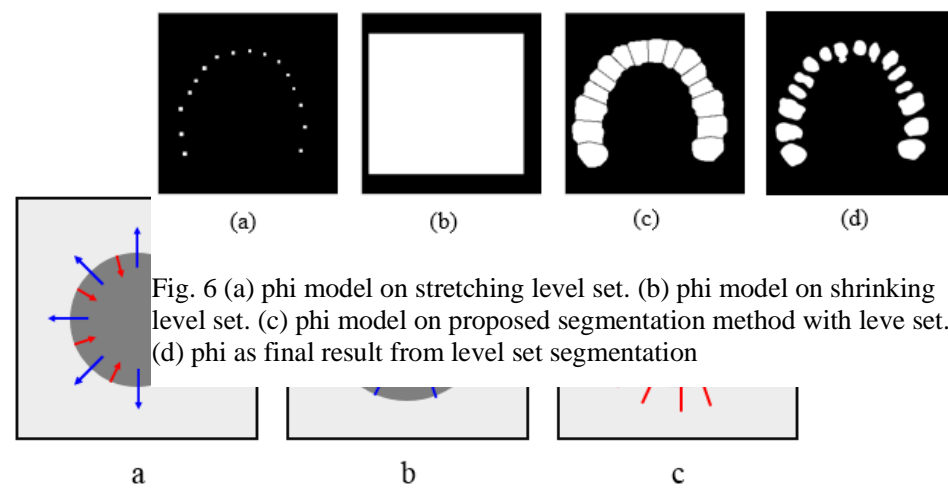

Fig. 5 (a) Gradient direction on the tooth object. (b) Gradient directions for one tooth object with a hole inside it. (c) Normal gradient directions of an object.

The first process is the edge indicator on the slice of CBCT image that is used to detect the edge of a dental object. Edge indicator can be defined as a positive and decreased function in terms of the image gradient for energy minimization problem. The formulation of this edge detector is as follows: 


$$
g(x, y)=\frac{1}{1+\left|\nabla I_{G}(x, y)\right|^{2}}
$$

where, $\nabla I_{g}=G_{\sigma}(x, y) * I(x, y)$, smoother version of $\mathrm{I}$, is convolution of the image $I$ with the Gaussian kernel of standard deviation $\sigma$.

The teeth in dental CBCT images present non-uniform levels and distribution of intensities due to the tooth structure consisting of various parts such as the pulp, dentine, and enamel. We implemented the gradient direction filter process to improve the contours of any of the intensity levels [21]. There are several gradient direction models that exist in CBCT images of teeth. Three gradient direction the teeth are shown as shown in Fig. 5. Fig. 5a shows the non-uniform direction of the gradient direction where there is a direction going into the object and going out away from the object. The direction the gradient exits from the object usually refers to other objects or areas that have high intensity. Fig. $5 \mathrm{~b}$ shows the non-uniform gradient direction out of the object due to a part of the tooth such as the pulp. Fig. $5 \mathrm{c}$ is the normal gradient direction of an object with the negative signed distance function.

The gradient direction function is implemented on the results of the edge indicator process. The tooth boundary on a valid edge indicator is a gradient that is consistent with the normal direction [14]. The formulation for this process is presented in Equation (8).

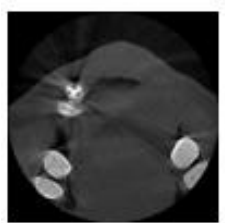

(1)

Fig. 7 Input image

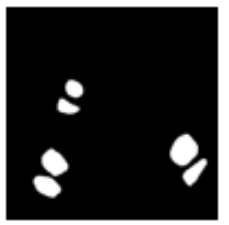

(1)

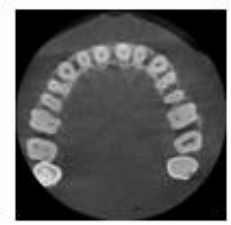

(2)

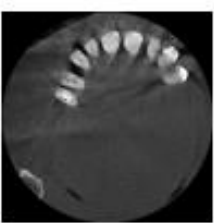

(3)

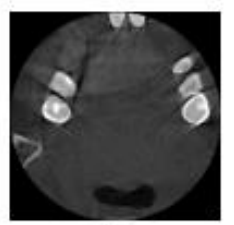

(4)

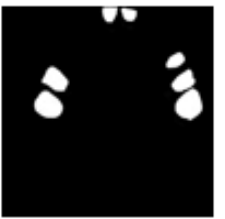

(4)

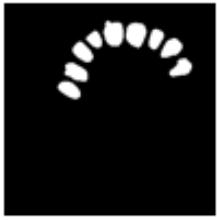

(3)

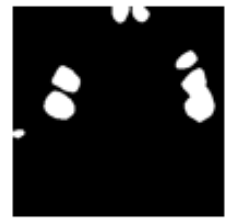

(4) where $\nabla \phi \cdot \nabla I_{g}$ is a comparison between the normal gradient direction and the test image gradient direction. The normal direction is designed from the dental ROI image with the core image of the tooth object that has been obtained. Broadly speaking, this equation serves to filter equation 7 for the edge detector of objects from dental CBCT images.

The final process is contour tracking using the level set method where the last ROI is used as the initial contour. The level set method, as an object form tracking method, can be categorized into two types. One of the distinguishing features of the two models is how the mark or phi value of the level set method is modeled. The first is the type of stretching level set where phi is modeled by marking a small rectangle area inside the object. Fig. 6a is an example of implementing the phi model of this type of level set. Each dental object is manually marked with a small rectangle. All of the signs are phi values from the level set method. The stretch with certain iteration goes to get the shape of the gear object. The second type is the shrinking set level where phi is modeled as the area that surrounds the object. Fig. 6b shows the phi model if implemented in the dental CBCT slice. Visually, inside large rectangular objects contained dental objects inside. It is nothing but a phi model that is depreciated by the level set method for tracking dental objects.

Fig. 8 Ground truth image

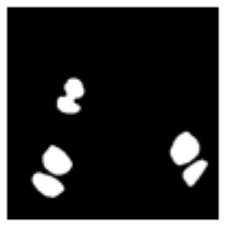

(1)

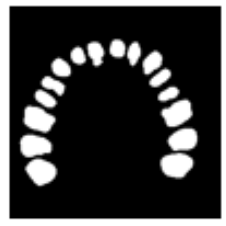

(2)
(3)

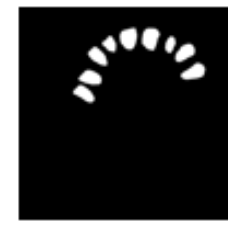

Fig. 9 Segmentation result with proposed method

$$
g=\left\{\begin{array}{r}
1, \nabla \phi \cdot \nabla I_{g} \leq 0 \\
\frac{1}{1+\left|\nabla I_{G}(x, y)\right|^{2}}, \quad \nabla \phi \cdot \nabla I_{g}>0
\end{array}\right.
$$

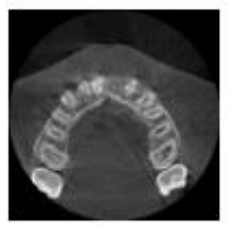

(5)

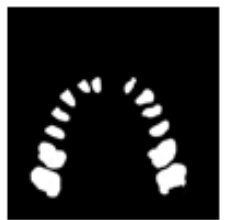

(5)

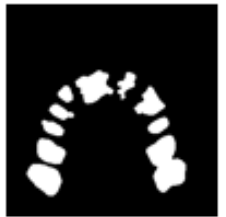

(5)

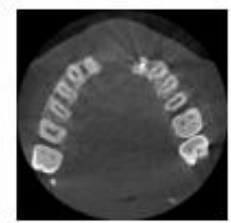

(6)

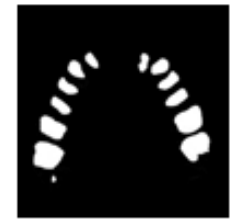

(6)

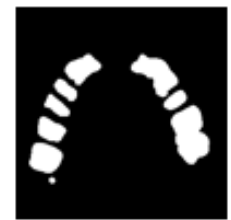

(6)

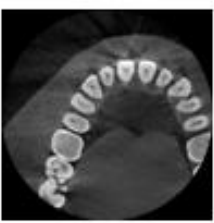

(7)

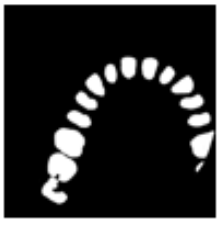

(7)

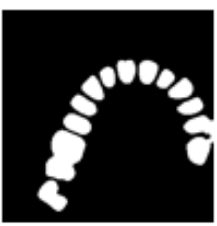

(7)
Fig. 6c is a phi model that is built and implemented into the proposed method. The block diagram of how this model was built is visualized in Fig. 2. The ROI estimation of each tooth object is used as the initial phi model of the level set 
method. This object tracking segmentation method is a type of shrink level set which is similar to the phi model in Fig. $6 \mathrm{~b}$. However, the model simplifies the shape of the initial scope. Visually, the object area is more focused on dental objects directly. The level set method is used to evolve the contour to get the actual shape of the tooth object being segmented to produce the results as Fig. 6d shows. The phi model built on the proposed method will minimize the cost of iteration time used to track dental objects. Fig. 2 shows the final result of the contour tracking segmentation process that previously shaped objects such as the ROI image that was evolved so that the actual shape of the dental object is captured in the CBCT slice image of the tooth.

\section{EXPERIMENT RESULTS}

The implementation of this method used Matlab software version R2018b with gray scale image data format obtained from the Dental and Oral Hospital, Airlangga University (RSGM UNAIR). The image data is a 3D CBCT image of 7 patients in which 10 subjects will be tested for dental slices. Each image is a CBCT dental grayscale slice with axial projection with dimensions of 266x266 pixels.

Fig. 6 shows an example of the CBCT dental slice image of each patient being tested. On the other hand, Fig. 7 is the image of its ground truth created with the help of experts in the field of dentistry. This image is used as comparison data to see the level of accuracy of the segmentation results as shown in Fig. 8.

Visually, the developed segmentation method is able to get dental objects. The similarity between the results of segmentation with the image of its ground truth proves the success of this method. However, the success of this method is not sufficient to be proven by visualizing the results of segmentation. Several experimental evaluation scenarios were used to prove the success rate of the method developed.

To evaluate the proposed method, the results of segmentation are compared with the ground truth data which is a manually segmentation dataset from an expert. In this case, the four-pixel types are calculated as follows:

- True-positive (TP): Correctly segmented as dental tissues.

- True-negative (TN): Correctly segmented as nondental tissues.

- False-positive (FP): Incorrectly segmented as dental tissues (teeth, enamel, and pulp).

- False-negative (FN): Incorrectly segmented as nondental tissues

The evaluation metrics are used in each image to evaluation between result and ground truth as follows:[22]

$$
\begin{gathered}
\text { Accuracy }=\frac{T P+T N}{\text { Number of Pixel }} \\
\text { Sensitivity }=\frac{T P}{T P+F N}
\end{gathered}
$$

$$
\text { Spesificity }=\frac{T N}{T N+F P}
$$

The accuracy parameter is used to see the quantity of success of the proposed method. However, due to the problem of unbalanced datasets, the accuracy parameter cannot be the only evaluation metric for measuring the performance of a method. A high degree of accuracy is not enough to show the merit of the method being evaluated. Therefore, sensitivity (positive rate) and specificity (negative rate) are also used to evaluate the performance of the proposed segmentation method.

Evaluation results between the results of the proposed methods and the ground truth image of each subject are shown as in Table I.

TABLE I RESULTS OF EXPERIMENTS FROM EACH PATIENT

\begin{tabular}{|c|c|c|c|}
\hline Patient & Accuracy & Sensitivity & Spesificity \\
\hline 1 & $97.80 \%$ & $88.09 \%$ & $98.18 \%$ \\
\hline 2 & $93.61 \%$ & $84.85 \%$ & $94.74 \%$ \\
\hline 3 & $94.79 \%$ & $76.55 \%$ & $96.21 \%$ \\
\hline 4 & $97.12 \%$ & $92.05 \%$ & $97.38 \%$ \\
\hline 5 & $94.83 \%$ & $73.02 \%$ & $96.53 \%$ \\
\hline 6 & $94.17 \%$ & $91.28 \%$ & $94.51 \%$ \\
\hline 7 & $95.60 \%$ & $91.66 \%$ & $96.06 \%$ \\
\hline
\end{tabular}

The evaluation values in Table 1 show a variety of different results. However, the average evaluation value obtained is above nine. This indicates that the method shown is able to get dental objects optimally. There is a sensitivity value that has a range of values below nine. The greatest accuracy was obtained in patient 1 with a value of $97.80 \%$. However, the most optimal results obtained are shown by the 4 th patient where if the values of the three parameters are averaged, it results in a higher value than the other patients. This proves that accuracy alone is not enough as an evaluation parameter of a method.

In the results table there is a sensitivity value there is a value below ninety percent. It can be caused by several factors such as the patient's dental data that uses braces in the scanning process so that a diffusion of light that disrupts the shape of the object appears.

Some slices of CBCT images do have factors that affect the results. Like a slice that represents the root of a tooth. In this section the object is tangent to the bone of the mouth. Between the dental intensity and bone have relatively the same values. Three are only the boundary of the object has a slight difference value. Therefore, our proposed method is based on the eval set method which focuses on generating edge values of objects.

The next experimental scenario in this paper is the comparison between the results of the method with several methods related to the segmentation of the level set method in dental CBCT images. Table II shows the results of 
comparing accuracy, sensitivity, and specifications with several methods.

TABLE II COMPARISON RESULT WITH OTHER METHOD

\begin{tabular}{|c|c|c|c|}
\hline Method & Acc. & Sen. & Spe. \\
\hline 3D Region Mergin [9] & $97.79 \%$ & $69.00 \%$ & $98.48 \%$ \\
\hline Deep Learning [6] & $94.26 \%$ & $90.09 \%$ & $94.07 \%$ \\
\hline Proposed Method & $95.51 \%$ & $87.91 \%$ & $96.14 \%$ \\
\hline
\end{tabular}

Comparative evaluation between the proposed method and the two comparison methods obtained the average value of accuracy, sensitivity, and specifications, respectively. The region merging method has a higher level of accuracy compared to the proposed method and the deep learning method. However, the effectiveness of a method is not only determined by one parameter. The sensitivity value of the merge method of a region has a significant difference than other methods. It shows that the accuracy for segmenting objects with the tooth method is good but other objects that have the same characteristics are also segmented. Other objects can be like bones in the framework of the mouth that tend to have the same intensity as dental objects.

The proposed method in general has a balance value with other parameters. This method has a future work that must be done to increase the value of evaluation. In general, the proposed method is capable of segmenting object contour tracking automatically by producing a fairly optimal level of accuracy. Methods can be developed to segment the entire slice based on this proposed method.

\section{CONCLUSION AND FUTURE WORK}

In this paper, we proposed an automatic segmentation for slice of dental computed tomography image. The proposed method which is based on a level set method with morphology operations to determine of the ROI area of the tooth structure and polynomial fittings to specify the area of the tooth object, works properly. From the experimental results, the automatic segmentation method that was designed was able to segment the dental objects optimally with accuracy, sensitivity, and specifications of $95.51 \%$, $87.91 \%$, and $96.14 \%$, respectively. In addition, the development of the phi model carried out in this proposed method can simplify the model used at the level set in general. There are several future works that can be done and we will work in the future. Among them is the improvement of the image of the patient's teeth using braces in the scanning process. The design of drawing improvement methods can be done to overcome this. Increased accuracy in images that have similar similarities between dental objects and other objects. Then the application of this method can be used to segment the entire slice directly.

\section{DAFTAR PUSTAKA}

[1] A. Nowakowski, Biomedical engineering handbook (Vol. 2). 2006.

[2] T. Kiljunen, T. Kaasalainen, A. Suomalainen, and M. Kortesniemi, "Dental cone beam CT: A review," Phys. Medica, vol. 31, no. 8, pp. 844-860, Dec. 2015.

[3] J. He, C.-S. Kim, and C.-C. J. Kuo, Interactive Segmentation Techniques Algorithms and Performance Evaluation. 2014.

[4] L. Wang et al., "Automated segmentation of dental CBCT image with prior-guided sequential random forests," 2016.

[5] M. E. Barrett WA1, "Interactive live-wire boundary extraction. - PubMed - NCBI," 1997. [Online]. Available: https://www.ncbi.nlm.nih.gov/pubmed/9873914.

[Accessed: 05-Aug-2019].

[6] R. Indraswari, T. Kurita, A. Z. Arifin, N. Suciati, and E. R. Astuti, "Multi-projection deep learning network for segmentation of 3D medical images," Pattern Recognit. Lett., vol. 125, pp. 791-797, Jul. 2019.

[7] R. Adams and L. Bischof, "Seeded region growing," IEEE Trans. Pattern Anal. Mach. Intell., vol. 16, no. 6, pp. 641647, Jun. 1994.

[8] A. K. Jain and A. K., Fundamentals of digital image processing. Prentice Hall, 1989.

[9] R. Indraswari, T. Kurita, A. Z. Arifin, N. Suciati, E. R. Astuti, and D. A. Navastara, "3D Region Merging for Segmentation of Teeth on Cone-Beam Computed Tomography Images," 2018 Jt. 10th Int. Conf. Soft Comput. Intell. Syst. 19th Int. Symp. Adv. Intell. Syst., pp. 341-345, 2018.

[10] R. Indraswari, A. Zainal Arifin, N. Suciati, E. Renwi Astuti, and T. Kurita, "Automatic Segmentation of Mandibular Cortical Bone on Cone-Beam CT Images Based on Histogram Thresholding and Polynomial Fitting," Int. J. Intell. Eng. Syst., vol. 12, no. 4.

[11] V. Kumar et al., "Radiomics: The process and the challenges," Magn. Reson. Imaging, vol. 30, no. 9, pp. 1234-1248, 2012.

[12] S. Osher and J. A. Sethian, "Fronts propagating with curvature-dependent speed: Algorithms based on Hamilton-Jacobi formulations," J. Comput. Phys., vol. 79, no. 1, pp. 12-49, Nov. 1988.

[13] Z. Xia, Y. Gan, L. Chang, J. Xiong, and Q. Zhao, "Individual tooth segmentation from CT images scanned with contacts of maxillary and mandible teeth," Comput. Methods Programs Biomed., vol. 138, pp. 1-12, Jan. 2017.

[14] H. Gao and O. Chae, "Individual tooth segmentation from CT images using level set method with shape and intensity prior," Pattern Recognit., vol. 43, no. 7, pp. 2406-2417, Jul. 2010

[15] D. X. Ji, S. H. Ong, and K. W. C. Foong, “A level-set based approach for anterior teeth segmentation in cone beam computed tomography images," Comput. Biol. Med., vol. 50, pp. 116-128, Jul. 2014.

[16] G. Y., X. Z., X. J., Z. Q., H. Y., and Z. J., "Toward accurate tooth segmentation from computed tomography images using a hybrid level set model," Med. Phys., vol. 42, no. 1, pp. 14-27, 2014.

[17] H.-T. Yau, T.-J. Yang, and Y.-C. Chen, "Tooth model reconstruction based upon data fusion for orthodontic treatment simulation," Comput. Biol. Med., vol. 48, pp. 8 16, May 2014.

[18] C. Li, C. Xu, C. Gui, and M. D. Fox, "Distance regularized level set evolution and its application to image 
segmentation," IEEE Trans. Image Process., vol. 19, no. 12, pp. 3243-3254, Dec. 2010.

[19] W. R. Gonzalez RC, "Digital Image Processing 2nd," New Jersey Tom Robbins;, 2002.

[20] A. Z. Arifin and A. Asano, "Image segmentation by histogram thresholding using hierarchical cluster analysis," Pattern Recognit. Lett., vol. 27, no. 13, pp. 1515-1521,
Oct. 2006.

[21] H. Gao and O. Chae, "Touching tooth segmentation from CT image sequences using coupled level set method," IET Conf. Publ., no. 543 CP, pp. 382-387, 2008.

[22] M. Sokolova and G. Lapalme, "A systematic analysis of performance measures for classification tasks," Inf. Process. Manag., vol. 45, no. 4, pp. 427-437, 2009. 